Article

\title{
Theoretical Model of the Axial Residual Velocity of PELE Projectiles Penetrating Thin Metal Targets
}

\author{
Liangliang Ding ${ }^{1}$, Wenhui Tang ${ }^{1, *} \mathbb{E}$, Xianwen $\operatorname{Ran}^{1, *}$, Zijian Fan $^{2}$ and Weike Chen ${ }^{3}$ \\ 1 College of Liberal Arts and Sciences, National University of Defense Technology, Changsha 410073, China; \\ dingliangliang14@nudt.edu.cn \\ 2 College of Aeronautics and Astronautics, National University of Defense Technology, Changsha 410073, \\ China; zjfan97@mail.ustc.edu.cn \\ 3 Minghua Machinery Limited Company of Guangdong, Foshan 528231, China; cwk821495@163.com \\ * Correspondence: tangwenhui@nudt.edu.cn (W.T.); ranxianwen@nudt.edu.cn (X.R.); \\ Tel.: +86-181-7512-1477 (W.T.); +86-135-7415-1246 (X.R.)
}

Received: 17 April 2019; Accepted: 6 June 2019; Published: 10 June 2019

\begin{abstract}
With the increase of battlefield target diversity and protection mobility, the disadvantages of traditional armor piercing warheads have gradually become prominent. The conception of the PELE (penetration with enhanced lateral efficiency) projectile was thus proposed. The axial residual velocity of the projectile is a very important indicator of a PELE projectile, which mainly reflects the penetration ability of the PELE projectile. The PELE projectile is a symmetrical structure, so the collision problem can be simplified to plane collision. Furthermore, the two-dimensional plane is axisymmetric, and so it can be further simplified to one-dimensional collision. Based on simplification and assumptions, the mechanism of a PELE projectile penetrating a thin metal target plate was studied using the shock wave theory, and a theoretical model of axial residual velocity has been established in this article. The energy loss during the penetration process was divided into the following parts: the kinetic energy increment of the target plug in the impact region, the internal energy increment of the outer casing and inner core, and the shear energy dissipation of the projectile against the target plate. In addition, the specific methods of determining the energy loss of each part are given in detail. According to the conservation of energy, the approximate calculation formulae of the axial residual velocity of a PELE projectile have been deduced. Finally, the theoretical results were compared with the experimental results under different working conditions, and the results were in good agreement. Therefore, the theoretical model has application value and guiding significance in the field of engineering.
\end{abstract}

Keywords: PELE projectile; axial residual velocity; penetration; shock wave theory; metal thin targets; symmetric simplification

\section{Introduction}

The PELE (penetration with enhanced lateral efficiency) projectile is a new type of penetrating body proposed in recent years. This new type of ammunition is the result of a cooperation between the French-German Research Institute Saint Louis, the GEKE Technology in Freiburg, and the Diehl Munitionssysteme. PELE projectiles have the advantage of being able to convert axial velocity into radial velocity without any pyrotechnical agents. They are mainly composed of a high density outer casing and a low density inner core; a simplified schematic diagram is shown in Figure 1. The outer casing is usually made of heavy metals such as steel or tungsten, which can achieve good penetration performance when impacting the metal target plate. The inner core is made of inert materials such as plastic or aluminum, which are relatively weak in penetration. When the PELE projectile impacts the 
metal target plate, the inner core moves slowly forward and is squeezed between the crater and outer casing. The rising pressure in the inner core causes the surrounding casing to expand and produce a radial force on the casing, as shown in Figure 2a. As the projectile perforates the target plate, the radial force of the inner core is released, and the outer casing is broken down into large numbers of fragments radially, as shown in Figure $2 b$. Therefore, the PELE projectile not only has stronger penetration ability, but also has stronger lateral damage performance $[1,2]$.

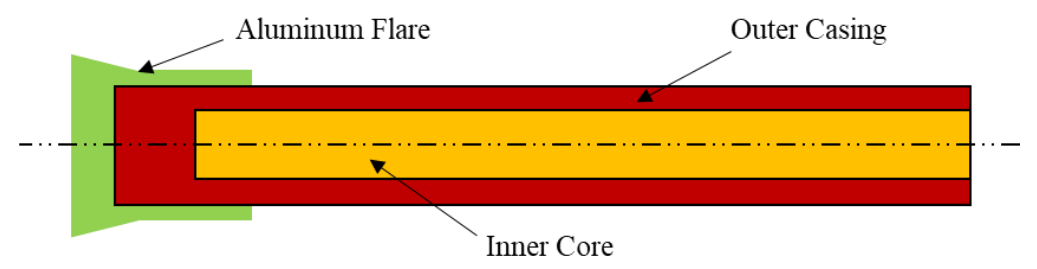

Figure 1. Longitudinal section of the simplified PELE (penetration with enhanced lateral efficiency) projectile.

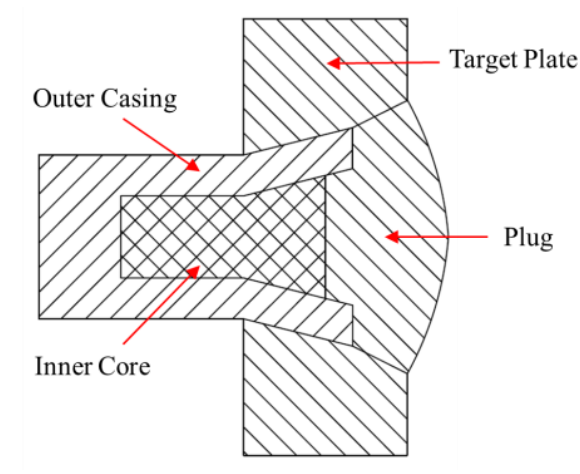

(a)

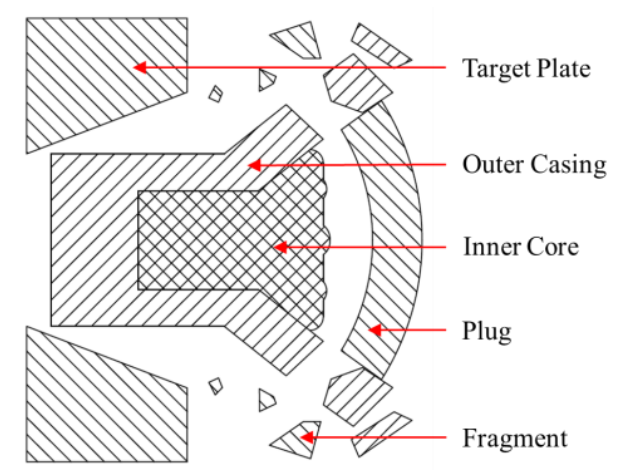

(b)

Figure 2. Different penetration states of the PELE projectile. (a) Initial penetration state; (b) completely perforating state.

The axial residual velocity of the projectile, the radial scattering velocity, and the spatial distribution of fragments are important indicators used to measure damage efficiency. Many experts and scholars [3-8] have studied the factors that affect the lateral effect of PELE projectiles, such as the impact velocity, the intersection posture between the projectile and target plate, the material of the inner core, and so on. However, experiments only reflect changes in state, which cannot give the specific changes in the process. In order to reach a clearer and more intuitive understanding of the penetration process of the PELE projectile and its mechanism of action, Paulus [3], Verreault [9], and other scholars [10-12] carried out a lot of numerical simulations of the penetration process of PELE projectiles using the finite element simulation software Autodyn (Century Dynamics, FortWorth, TX, USA) and LS-DYNA (Livermore Software Technology Corporation, Livermore, CA, USA), and obtained the key factors that affect the damage power of PELE projectiles. Neither experiments nor simulations can describe the penetration process of PELE projectiles theoretically. By using elastic wave theory and energy conservation, Paulus [3] established the calculation model of the axial residual velocity and the radial scattering velocity after PELE projectile penetrating of a target plate. Zhu [13] and $\mathrm{Du}$ [14] analyzed from experimental and theoretical perspectives the fragmentation effect of PELE after penetrating a metal target plate. However, the theoretical analysis models they built adopted the acoustic approximation, ignoring the multiple wave interactions at the inner core/target interface. According to shock wave theory and Motto fragment distribution theory $[15,16]$, Verreault $[9,17,18]$ established the theoretical model of the radial scattering velocity after PELE projectile penetrating of a 
target plate. Fan [19] developed a two-stage to estimate the radial velocity of PELE projectiles after perforating the target plate by using the shock wave theory.

Based on the above literature investigation, it can be found that most of the previous works on the penetration process of PELE projectiles have focused on experiments and numerical simulations. Moreover, there are some limitations in the theoretical analysis when using one-dimensional linear elastic wave theory to analyze the penetration process of PELE projectiles. The residual velocity of the projectile, the radial scattering velocity, and the spatial distribution of fragments are three important aspects of PELE projectiles. The residual velocity of the projectile mainly reflects the penetration ability of the PELE projectile. Although PELE projectiles are usually aimed at thinner targets, they are often used as a penetrating body to penetrate thicker targets or even multi-layer targets, whereupon the projectile velocity will also be greatly attenuated. Therefore, it is necessary to establish a theoretical model of the residual velocity of a projectile with a wider range of speeds. In view of this, the main research work of this paper was based on shock wave theory and energy conversion; the energy dissipation during the penetration process were analyzed in detail. On this basis, the theoretical model of axial residual velocity of a PELE projectile penetrating a metal thin target plate was established, and the theoretical model was validated by experiments.

\section{Establishment of the Theoretical Model of Axial Residual Velocity}

\subsection{Plane Shock Wave Assumption of PELE Projectile Penetrating Against Target Plate}

Since the PELE projectile has a symmetrical structure, the collision between the projectile and the target plate can be simplified to a plane collision problem. When the PELE projectile impacts the target plate, two shock waves are generated at the contact interface between the projectile and target plate. The shock waves propagate to the rear of the projectile and the target plate respectively, as shown in Figure 3a. Since the target thickness is much smaller than the projectile length, the shock waves in the target plate first reach the free interface of the target plate and reflect rarefaction waves to the contact interface, as shown in Figure 3b. In order to facilitate the division of the penetration process, the following definitions are made: $t_{0}$ represents the moment when the projectile has just touched the target plate; $t_{1}$ represents the moment when the rarefaction waves from the back side of the target plate reach the contact interface; $t_{2}$ represents the moment when the PELE projectile just perforates the target plate. The time range $t_{0}-t_{1}$ is defined as the impact stage, and the time range $t_{1}-t_{2}$ is defined as the plug shear stage. Thus, the process of the PELE projectile penetrating the target plate can be divided into two stages: projectile impact stage and plug shear stage.

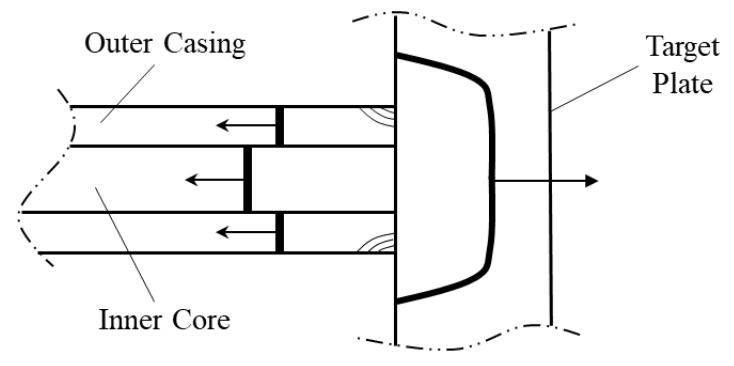

(a)

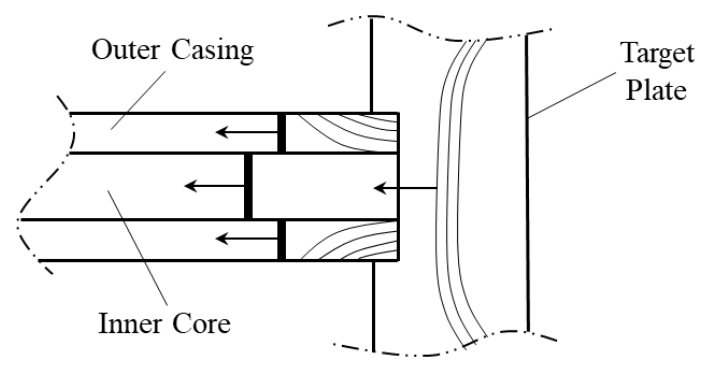

(b)

Figure 3. Propagating schematic diagram of shock waves and rarefaction waves upon PELE projectile impacting the target plate. (a) Shock waves propagate in the projectile and target plate; (b) rarefaction waves propagate in the projectile and target plate.

On the moment of impact between the PELE projectile and target plate, one shock wave is produced in the target plate and propagates to the back of the target plate, and another shock wave, opposite to the projectile's flight direction, is generated in the projectile. Due to the inner core material 
being different from the outer casing, the intensity and shock wave velocity of the inner core and outer casing are different. After the plane shock wave, the rarefaction wave propagates radially from the interface to the projectile interior, which causes the projectile to expand radially. The plane shock wave relationship is obtained under the one-dimensional stress condition. To analyze the process of a PELE penetrating a target plate using the plane shock wave theory, the following assumptions are required:

(1) Neglecting the influence of shearing force on the impact wave intensity. The shearing force is caused by the difference of particle velocity between the projectile and target plate, which makes the impact velocity decrease and the impact pressure increase. For a thin target plate, the shearing force is smaller than the impact pressure, so it can be neglected.

(2) The radial expansion of projectile is neglected before the projectile perforates the target plate. After the shock wave, the projectile casing will expand radially under the action of the rarefaction wave. However, within the time range of investigation, the radial expansion value of the projectile is limited, and it is considered that the radial deformation of the outer casing is limited by the target plate. Therefore, the radial expansion of the projectile is neglected in the perforation process.

Since both the PELE projectile and the target plate have symmetrical structures, when analyzing the stress state of the projectile and the target plate, it can be simplified into a 2D (two-dimensional) plane problem and the $2 \mathrm{D}$ plane is axisymmetric. In this paper, the axial residual velocity of the projectile is mainly studied, not the radial dispersion of fragments after the projectile perforates the target plate. Therefore, combined with the above two basic assumptions, the study of the axial residual velocity of projectile can be further simplified to a 1D (one-dimensional) collision problem. The penetration process of the PELE projectile against a thin metal target plate is similar to that of the flat head projectile, and the penetration process can be analyzed using the shear resistance model of the flat head projectile. The shear resistance model divides the energy loss during the penetration process into two parts: the impact energy dissipation between the projectile and the target plate, and the shear energy dissipation of the plug in the perforation process.

According to shock wave theory, the impact energy dissipation can be divided into the following parts: impact compression potential energy of the plug, the kinetic energy of particles after the shock wave, and the impact compression potential energy of the outer casing and inner core etc. Based on the characteristics of the PELE projectile, the shear energy dissipation of the plug is divided separately into the inner edge and outer edge of projectile casing shear of the target plate.

\subsection{Internal Energy and Kinetic Energy Increment of the Target Plate Plug after Shock Waves}

Before the rarefaction wave reflects from the back side of the target plate, the impact between the projectile and the target plate can be approximately considered a coaxial impact problem, as shown in Figure 4. The projectile comprises two parts, outer casing and inner core, and their internal shock wave intensities are different because the materials are different. Correspondingly, the shock wave intensities in different impact regions of the target plate are different.

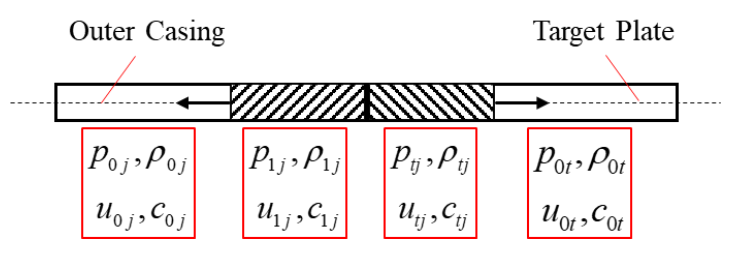

(a)

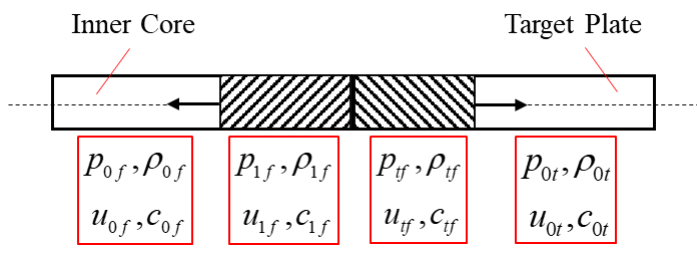

(b)

Figure 4. Simplified schematic diagram of the interaction between projectile and target plate. (a) Interaction between outer casing and target plate; (b) interaction between inner core and target plate.

It is assumed that then the projectile impacts the target plate with the mass $m$ and velocity $u_{0}$, the initial velocity and pressure of the target plate and the initial pressure of the projectile are both 
zero. Here, the following definitions are made. $P$ stands for the pressure per unit area, $\rho$ is the material density, $D$ represents the shock wave velocity, $u$ is the particle velocity, $E$ means the energy, $c$, and $\lambda$ represents the Hugoniot constant of the material. In addition, the subscript $j$ is an abbreviation for "jacket," and it is used to denote the parameters of the projectile outer casing material; the subscript $f$ is an abbreviation for "filling," and it is used to denote the parameters of the inner core material; the subscript $t$ represents the parameters of the target plate, and it is an abbreviation for "target." The subscript $t j$ represents the post-wave state of target plate after the collision between the outer casing and target plate, and the subscript $t f$ represents the post-wave state of the target plate after the collision between the inner core and target plate. The subscript 0 indicates the wave-front state of the material, and the subscript 1 indicates the post-wave state of the material. According to the interaction of the shock wave, the following relationships can be obtained.

(1) Take the projectile outer casing as the main body of research. The initial state of the outer casing is $P_{0 j}, \rho_{0 j}, u_{0 j}, c_{0 j}$, and a left shock wave is generated in the outer casing after the impact, and the post-wave state is $P_{1 j}, \rho_{1 j}, u_{1 j}, c_{1 j}$. Thus, the following relationships can be obtained through the shock wave discontinuous equation and the linear shock equation.

$$
\begin{gathered}
P_{1 j}=\rho_{0 j}\left(D_{j}-u_{0}\right)\left(u_{1 j}-u_{0}\right) \\
D_{j}-u_{0}=-c_{0 j}+\lambda_{j}\left(u_{1 j}-u_{0}\right)
\end{gathered}
$$

(2) Take the projectile inner core as the main body of research. The initial state of the inner core is $P_{0 f}, \rho_{0 f}, u_{0 f}, c_{0 f}$, and a left shock wave is generated in the inner core after the impact, and the post-wave state is $P_{1 f}, \rho_{1 f}, u_{1 f}, c_{1 f}$. Similarly, the following relationships can be obtained by the shock wave discontinuous equation and the linear shock equation.

$$
\begin{gathered}
P_{1 f}=\rho_{0 f}\left(D_{f}-u_{0}\right)\left(u_{1 f}-u_{0}\right) \\
D_{f}-u_{0}=-c_{0 f}+\lambda_{f}\left(u_{1 f}-u_{0}\right)
\end{gathered}
$$

(3) Take the target plate as the main body of research. The initial state of the target plate is $P_{0 t}, \rho_{0 t}$, $u_{0 t}, c_{0 t}$, and a right shock wave is generated in the target plate after the impact. The post-wave state is divided into two regions, as follows.

(a) The interaction region between the outer casing and the target plate. In this case, the corresponding post-wave state is $P_{t j}, \rho_{t j}, u_{t j}, c_{t j}$. Similarly, the following relationships can be obtained through the shock wave discontinuous equation and the linear shock equation.

$$
\begin{gathered}
P_{t j}=\rho_{0 t} D_{t j} u_{t j} \\
D_{t j}=c_{0 t}+\lambda_{t} u_{t j}
\end{gathered}
$$

(b) The interaction region between the inner core and the target plate. In this case, the corresponding post-wave state is $P_{t f}, \rho_{t f}, u_{t f}, c_{t f}$. Similarly, the following relationships can be obtained through the shock wave discontinuous equation and the linear shock equation.

$$
\begin{gathered}
P_{t f}=\rho_{0 t} D_{t f} u_{t f} \\
D_{t f}=c_{0 t}+\lambda_{t} u_{t f}
\end{gathered}
$$

According to the mechanical equilibrium conditions of the interaction region, the velocity and pressure on both sides of the discontinuity are equal. Hence, the following relationships can be obtained. 
(a) The balance condition between the outer casing and the target plate, $P_{t j}=P_{1 j}, u_{t j}=u_{1 j}$. When Equations (1), (2), (5), and (6) are combined together, the expression of the particle velocity of the target plate after the shock wave is as follows.

$$
\left(\rho_{0 j} \lambda_{j}-\rho_{0 t} \lambda_{t}\right) u_{t j}^{2}-\left(2 \rho_{0 j} \lambda_{j} u_{0}+\rho_{0 j} c_{0 j}+\rho_{0 t} c_{0 t}\right) u_{t j}+\rho_{0 j} \lambda_{j} u_{0}^{2}+\rho_{0 j} c_{0 j} u_{0}=0
$$

By solving Equation (9), the particle velocity after the impact between the outer casing and the target plate can be obtained.

$$
u_{t j}=\frac{\left(2 \rho_{0 j} \lambda_{j} u_{0}+\rho_{0 j} c_{0 j}+\rho_{0 t} c_{0 t}\right)-\sqrt{\left(2 \rho_{0 j} \lambda_{j} u_{0}+\rho_{0 j} c_{0 j}+\rho_{0 t} c_{0 t}\right)^{2}-4\left(\rho_{0 j} \lambda_{j}-\rho_{0 t} \lambda_{t}\right)\left(\rho_{0 j} \lambda_{j} u_{0}^{2}+\rho_{0 j} c_{0 j} u_{0}\right)}}{2\left(\rho_{0 j} \lambda_{j}-\rho_{0 t} \lambda_{t}\right)}
$$

(b) The balance condition between the inner core and the target plate, $P_{t f}=P_{1 f}, u_{t f}=u_{1 f}$. When Equations (3), (4), (7), and (8) are combined together, the particle velocity after the impact between the inner core and the target plate can be obtained as follows.

$$
u_{t f}=\frac{\left(2 \rho_{0 f} \lambda_{f} u_{0}+\rho_{0 f} c_{0 f}+\rho_{0 t} c_{0 t}\right)-\sqrt{\left(2 \rho_{0 f} \lambda_{f} u_{0}+\rho_{0 f} c_{0 f}+\rho_{0 t} c_{0 t}\right)^{2}-4\left(\rho_{0 f} \lambda_{f}-\rho_{0 t} \lambda_{t}\right)\left(\rho_{0 f} \lambda_{f} u_{0}^{2}+\rho_{0 f} c_{0 f} u_{0}\right)}}{2\left(\rho_{0 f} \lambda_{f}-\rho_{0 t} \lambda_{t}\right)}
$$

Thus, the kinetic energy increase of the target plate in the impact region of the projectile and the target plate can be obtained after the shock wave.

$$
\begin{gathered}
E_{t j}=\frac{1}{2} \pi\left(R^{2}-r^{2}\right) h \rho_{0 t} u_{t j}^{2} \\
E_{t f}=\frac{1}{2} \pi r^{2} h \rho_{0 t} u_{t f}^{2}
\end{gathered}
$$

where $R$ is the projectile outer casing radius, $r$ is the inner core radius, and $h$ is the target plate thickness.

Under the experimental conditions, the projectile and the target plate system are at normal pressure and normal temperature, and the shock wave pressure formed by the impact process is relatively high, $P>>P_{0}$. The relation between the material pressure and the specific volume under the impact adiabatic condition is shown in Figure 5. The relationship $P>>P_{0}$ is reflected in Figure 5 where point $A$ is very close to point $M$, which means that the $A B$ line can be considered to be the diagonal of the rectangular $M N B C$. Under this state, the influence of the initial pressure can be neglected, and it can be assumed that the total work of the shock compression is evenly distributed between the internal energy and kinetic energy.

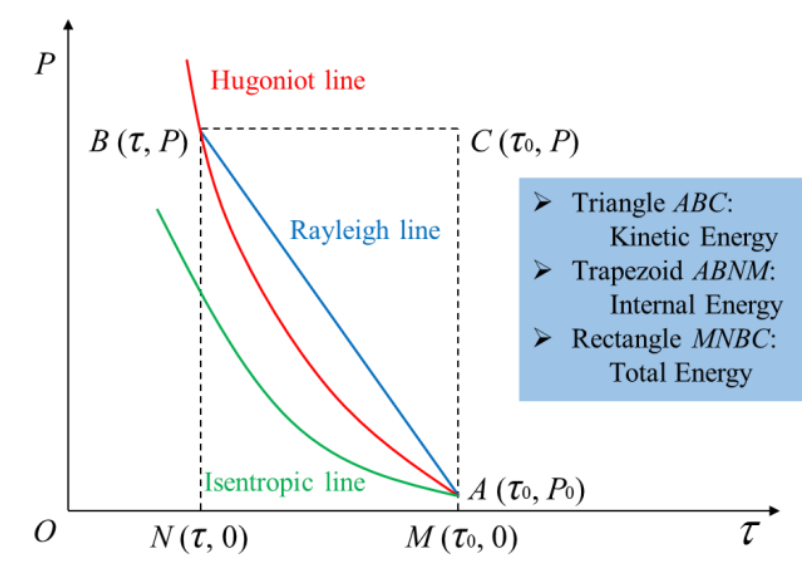

Figure 5. Distribution diagram of the shock wave energy. 


\subsection{Internal Energy Increment of Projectile after Shock Waves}

Combined with the shock wave relationship, the shock wave velocity $D_{t j}, D_{t f}, D_{j}$, and $D_{f}$ can be obtained. Where $D_{t j}$ represents the shock wave velocity of target plate under the impact between outer casing and target plate, $D_{t f}$ represents the impact wave velocity of target plate under the impact between inner core and target plate, and $D_{j}$ and $D_{f}$ represent the shock wave velocity of outer casing and inner core, respectively. The duration of the shock wave between the projectile and target plate is determined by the time from the projectile impacting the target plate to the rarefaction wave reaching the impact interface. It is assumed that the shock wave intensity unloading of the impact interface is completed once, and then the shock wave pressure duration $t_{j}$ and $t_{f}$ of the outer casing and the inner core can be expressed as follows.

$$
\begin{aligned}
& t_{j}=\frac{h}{D_{t j}}+\frac{\left(h-u_{t j} t_{j}\right)}{c_{0 t}-u_{t j}} \\
& t_{f}=\frac{h}{D_{t f}}+\frac{\left(h-u_{t f} t_{f}\right)}{c_{0 t}-u_{t f}}
\end{aligned}
$$

According to the Hugoniot relations, combined with the particle velocity of target plate after the shock wave, the internal energy change of the outer casing and the inner core during $t_{j}$ and $t_{f}$ can be expressed by the kinetic energy change.

$$
\begin{gathered}
E_{1 j}=\frac{1}{2} u_{1 j}^{2} \pi\left(R^{2}-r^{2}\right) D_{j} t_{j} \rho_{0 j} \\
E_{1 f}=\frac{1}{2} u_{1 f}^{2} \pi r^{2} D_{f} t_{f} \rho_{0 f}
\end{gathered}
$$

\subsection{Shear Energy Dissipation of the Target Plate Plug}

Since the shock wave impedance of the outer casing is higher than that of the inner core, the velocity of the annular contact interface between the outer casing and target plate is faster than that of the contact interface between the inner core and target plate during the penetration process. The working distance of the shear force is determined by the velocity difference and the movement time of the two contact interfaces.

It is assumed that the velocities of all the particles on the impact interface are always the same before the rarefaction wave propagates to the impact contact interface, and so the distance difference $\Delta h$ between the two interfaces during this period can be expressed as follows.

$$
\Delta h=\left(u_{t j}-u_{t f}\right) t_{f}
$$

According to the calculation theory of a flat head projectile penetrating a target plate [20], the shear energy dissipation of the outer edge of outer casing on the target plate $E_{\tau j}$ and that of the inner edge of outer casing on the target plate $E_{\tau f}$ can be expressed as follows.

$$
\begin{aligned}
& E_{\tau j}=\frac{1}{\sqrt{3}} \pi R \sigma_{Y}^{D} h^{2} \\
& E_{\tau f}=\frac{1}{\sqrt{3}} \pi r \sigma_{Y}^{D} \Delta h^{2}
\end{aligned}
$$

\subsection{Axial Residual Velocity of PELE Projectile after Perforating Against the Target Plate}

It is assumed that the internal energy increment of projectile by the shock wave is completely dissipated in the process of plastic deformation and rupture of the casing material and the kinetic energy of fragments, and cannot be converted into the kinetic energy of projectile again. In addition, 
the energy increment of the target plate plug under the action of the shock wave and the shear energy dissipation of the projectile on the target plate are both the loss of the kinetic energy of projectile. Since the target metal thickness is very thin, the mass loss of the PELE projectile can be neglected during the penetration process, and the mass of the residual projectile is approximately the same as the initial projectile mass, namely $m_{0}=m_{\text {res }}$. Therefore, the axial residual velocity of projectile after perforating against the target plate can be determined by the principle of energy conservation.

$$
\begin{gathered}
E_{0}=E_{r e s}+E_{t j}+E_{t f}+E_{1 j}+E_{1 t}+E_{\tau j}+E_{t f} \\
u_{\text {res }}=\sqrt{u_{0}^{2}-\frac{2}{m_{0}}\left(E_{t j}+E_{t f}+E_{1 j}+E_{1 t}+E_{\tau j}+E_{t f}\right)}
\end{gathered}
$$

\section{Verification and Analysis of the Theoretical Model}

\subsection{Literature Verification and Analysis of the Theoretical Model}

In Reference [3], the residual velocity of PELE projectiles with different inner cores penetrating metal thin target plates was measured. In the reference, the PELE projectiles were divided into two groups. The first group had an impact velocity less than $2500 \mathrm{~m} / \mathrm{s}$, total length of projectile of $50 \mathrm{~mm}$, projectile diameter of $10 \mathrm{~mm}$, and inner core diameter of $6 \mathrm{~mm}$. The second group had an impact velocity higher than $2500 \mathrm{~m} / \mathrm{s}$, total length of projectile of $40 \mathrm{~mm}$, projectile diameter of $8 \mathrm{~mm}$, and inner core diameter of $4 \mathrm{~mm}$. The outer casing materials in the two groups were tungsten alloy (D180K), and the inner core materials used were aluminum (A-G3) and polyethylene (PE) material. The target plates were made of aluminum (A-U4G) with $3 \mathrm{~mm}$ and $8 \mathrm{~mm}$ thickness, and steel target (XC48) with $3 \mathrm{~mm}$ thickness. The performance parameters of the projectiles and target plates are shown in Table 1 .

Table 1. Material properties of project and target plate $[3,21]$.

\begin{tabular}{lccccc}
\hline \multirow{2}{*}{ Material Properties } & Outer Casing Material & \multicolumn{2}{c}{ Inner Core Material } & \multicolumn{2}{c}{ Target Plate Material } \\
\cline { 2 - 6 } & D180K & A-G3 & PE & A-U4G & XC48 \\
\hline Density $\rho_{0}\left(\mathrm{~g} / \mathrm{cm}^{3}\right)$ & 18.0 & 2.65 & 0.92 & 2.8 & 7.82 \\
Sound velocity $c_{0}(\mathrm{~m} / \mathrm{s})$ & 4029 & 5176 & 2187 & 5106 & 4797 \\
Hugoniot constant $\lambda$ & 1.24 & 1.35 & 1.48 & 1.35 & 1.49 \\
Dynamic yield stress $\sigma_{\mathrm{y}} \mathrm{D}(\mathrm{Gpa})$ & - & - & - & 1.16 & 2.16 \\
\hline
\end{tabular}

Based on the above established theoretical model of axial residual velocity and the material parameters, the residual velocity of the PELE projectile under different conditions can be obtained. The comparison between the calculated results and the experimental results are shown in Tables 2 and 3, respectively. In the comparison verification, all the parameters of the model established in this paper are derived from Reference [3], not from self-measurement. The measurements in Reference [3] do not give the uncertainty. Paulus's calculation results [3] also do not take into account the uncertainty, directly substituting. In order to maintain the same significant digits as Paulus's calculation results, the calculation results of this paper also follow the idea of directly substituting all the parameters into the solution, and then rounding off and retaining the significant digits to units. It is worth noting that this article follows the principle of significant figure retention in the intermediate calculation process. 
Table 2. Residual velocity of experiment and calculation for A-G3 core material.

\begin{tabular}{|c|c|c|c|c|c|}
\hline \multicolumn{2}{|c|}{ Target Plate } & \multirow{2}{*}{$\begin{array}{l}\text { Experimental } \\
\text { Initial Velocity } \\
(\mathrm{m} / \mathrm{s})\end{array}$} & \multirow{2}{*}{$\begin{array}{l}\text { Experimental } \\
\text { Residual } \\
\text { Velocity }(\mathrm{m} / \mathrm{s})\end{array}$} & \multirow{2}{*}{$\begin{array}{l}\text { Calculated Residual } \\
\text { Velocity in } \\
\text { Reference [3] (m/s) }\end{array}$} & \multirow{2}{*}{$\begin{array}{c}\text { Calculated } \\
\text { Residual Velocity } \\
\text { in this Paper }(\mathrm{m} / \mathrm{s})\end{array}$} \\
\hline Material & $\begin{array}{c}\text { Thickness } \\
\text { (mm) }\end{array}$ & & & & \\
\hline \multirow{7}{*}{ A-U4G } & \multirow{3}{*}{3} & 929 & 914 & 916 & 912 \\
\hline & & 1275 & 1261 & 1257 & 1264 \\
\hline & & 2457 & 2444 & 2424 & 2436 \\
\hline & \multirow{4}{*}{8} & 937 & 900 & 898 & 902 \\
\hline & & 1254 & 1208 & 1206 & 1210 \\
\hline & & 2472 & 2434 & 2384 & 2416 \\
\hline & & 2984 & 2945 & - & 2907 \\
\hline \multirow{3}{*}{ XC48 } & \multirow{3}{*}{3} & 925 & 895 & 890 & 900 \\
\hline & & 1261 & 1231 & 1214 & 1237 \\
\hline & & 2441 & 2423 & 2352 & 2403 \\
\hline
\end{tabular}

Table 3. Residual velocity of experiment and calculation for PE core material.

\begin{tabular}{|c|c|c|c|c|c|}
\hline \multicolumn{2}{|c|}{ Target Plate } & \multirow{2}{*}{$\begin{array}{c}\text { Experimental } \\
\text { Initial Velocity } \\
(\mathrm{m} / \mathrm{s})\end{array}$} & \multirow{2}{*}{$\begin{array}{l}\text { Experimental } \\
\text { Residual } \\
\text { Velocity }(\mathrm{m} / \mathrm{s})\end{array}$} & \multirow{2}{*}{$\begin{array}{l}\text { Calculated Residual } \\
\text { Velocity in } \\
\text { Reference [3] (m/s) }\end{array}$} & \multirow{2}{*}{$\begin{array}{c}\text { Calculated } \\
\text { Residual Velocity } \\
\text { in this Paper }(\mathrm{m} / \mathrm{s})\end{array}$} \\
\hline Material & $\begin{array}{c}\text { Thickness } \\
(\mathrm{mm})\end{array}$ & & & & \\
\hline \multirow{7}{*}{ A-U4G } & \multirow{3}{*}{3} & 924 & 918 & 910 & 914 \\
\hline & & 1279 & 1263 & 1260 & 1266 \\
\hline & & 2420 & 2394 & 2386 & 2401 \\
\hline & \multirow{4}{*}{8} & 939 & 887 & 898 & 894 \\
\hline & & 1258 & 1203 & 1207 & 1200 \\
\hline & & 2445 & 2393 & 2353 & 2389 \\
\hline & & 2977 & 2952 & - & 2934 \\
\hline \multirow{3}{*}{ XC48 } & \multirow{3}{*}{3} & 936 & 889 & 899 & 897 \\
\hline & & 1262 & 1206 & 1213 & 1211 \\
\hline & & 2475 & 2462 & 2380 & 2436 \\
\hline
\end{tabular}

Paulus [3] assumed that weak shock waves will be generated in the PELE projectile when it impacts the target plate. Combined with the Recht-Ipson model and the Mott model, the residual velocity of projectile, the radial scattering velocity and the spatial distribution of fragments were then obtained. To make a more intuitive comparative analysis, the results calculated by the theoretical model in this paper are compared with those calculated by the theoretical model in Reference [3], taking the experimental results in Reference [3] as reference data. In order to characterize the degree of difference between the calculated results and the experimental results, we need to analyze the relative error, and the relative error $\alpha$ is defined as follow.

$$
\alpha=\frac{\left(u_{\text {ref }}-u_{\text {exp }}\right) \operatorname{or}\left(u_{\text {cal }}-u_{\text {exp }}\right)}{u_{\text {exp }}} \times 100 \%
$$

where $u_{r e f}$ represents the calculation result corresponding to the model established in Reference [3], $u_{c a l}$ represents the calculation result corresponding to the model established in this paper, and $u_{\text {exp }}$ represents the experimental result in Reference [3].

When using the relative error to compare the model established in this paper with that in Reference [3], they must be under the same initial conditions. Based on the relative error, the relationship between the residual velocity and the impact velocity of a projectile penetrating a target plate under different working conditions can be obtained intuitively, as shown in Figure 6. From the comparison of the experimental results in Reference [3] and the calculated results in Table 2, Table 3, and Figure 6, the calculated results obtained by the theoretical model of axial residual velocity in this paper are in good agreement with the measured experimental results in Reference [3]. 


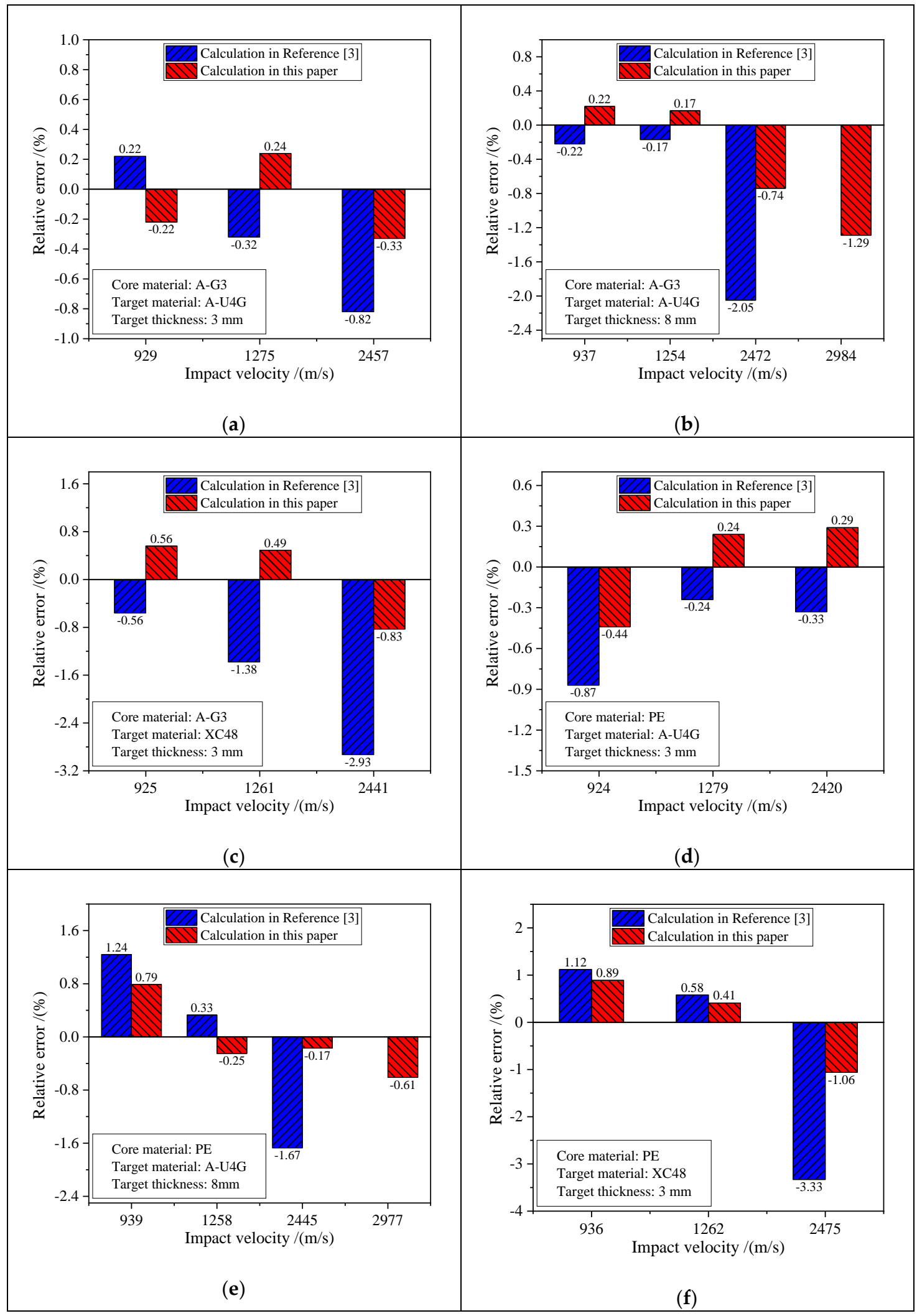

Figure 6. Comparison of residual velocity between reference results and calculation results. (a) A-G3 core, A-U4G target plate ( $3 \mathrm{~mm})$; (b) A-G3 core, A-U4G target plate $(8 \mathrm{~mm})$; (c) A-G3 core, XC48 target plate (3 mm); (d) PE core, A-U4G target plate (3 mm); (e) PE core, A-U4G target plate $(8 \mathrm{~mm})$; (f) PE core, XC48 target plate $(3 \mathrm{~mm})$. 
In addition, it was found that the calculated results based on the elastic wave theory used in Reference [3] are close to the experimental results at lower velocity. When the impact velocity is higher than $2500 \mathrm{~m} / \mathrm{s}$, elastic wave theory is not suitable for penetration calculation of PELE projectiles, and so the calculation results were not given in Reference [3]. However, the theoretical model established in this paper can be well matched for both lower and higher velocities. Since the theoretical model established in Reference [3] is based on elastic wave theory, the premise is that the deformation of the projectile and target should be subject to Hooke's law, which determines that the model is more suitable for the low-speed collision. When the impact velocity is high, the dynamic pressure generated by the projectile during the penetration process is very high, and the projectile and the target plate will undergo large deformation. At this point, the elastic moduli of the projectile and target material will no longer be constant, but will become a function of stress or strain. Therefore, there are limitations in using elastic wave theory to analyze the penetration process of projectile and target. This paper uses one-dimensional shock wave theory to analyze the penetration process of projectile and target, which can solve this problem well. Obviously, the calculated results based on shock wave theory in this paper are better than that based on the elastic wave theory in Reference [3], especially in high velocity conditions. The above comparative analysis can further confirm the rationality of the theoretical model established in this paper.

\subsection{Experiment Verification and Analysis of the Theoretical Model}

To further verify the rationality of the theoretical model established in this paper, two groups of experiments were designed and the axial residual velocities of projectiles were measured experimentally. In the experiments, the material of outer casing was tungsten alloy, and the length of projectile was $40 \mathrm{~mm}$, the inner radius was $3 \mathrm{~mm}$, the outer radius was $5 \mathrm{~mm}$, and the depth of inner hole was $37 \mathrm{~mm}$. The materials of the inner core were $\mathrm{Al}(6061 \mathrm{Al})$ and PTFE, with a radius of $3 \mathrm{~mm}$ and a height of $37 \mathrm{~mm}$. The material of target plate was Al (2024Al), with a thickness of $3 \mathrm{~mm}$. The material parameters of projectile and target plate are shown in Table 4.

Table 4. Material properties of project and target plate [3,22].

\begin{tabular}{lcccc}
\hline \multirow{2}{*}{ Material Properties } & Outer Casing Material & \multicolumn{2}{c}{ Inner Core Material } & Target Plate Material \\
\cline { 2 - 5 } & Tungsten & 6061Al & PTFE & 2024Al \\
\hline Density $\rho_{0}\left(\mathrm{~g} / \mathrm{cm}^{3}\right)$ & 18.0 & 2.7 & 2.15 & 2.8 \\
Sound velocity $c_{0}(\mathrm{~m} / \mathrm{s})$ & 4029 & 5350 & 1682 & 5370 \\
Hugoniot constant $\lambda$ & 1.24 & 1.34 & 1.82 & 1.3 \\
Dynamic yield stress $\sigma_{\mathrm{y}} \mathrm{D}(\mathrm{Gpa})$ & - & - & - & 1.2 \\
\hline
\end{tabular}

The projectile was wrapped with a layer of $1.5 \mathrm{~mm}$ thick copper, to ensure close integration between the experimental projectiles and launch gun rifles. The overall projectile is shown in Figure 7a, and the experimental launch gun is shown in Figure $7 \mathrm{~b}$. To measure the impact velocity of projectile, a light curtain was placed between the target plate and the launch gun, as shown in Figure 7c.

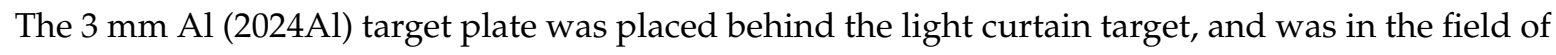
view of the high-speed photography. In addition, two copper foil targets were placed behind the target plate to test the residual axial velocity of the projectile, as shown in Figure $7 \mathrm{~d}$. In order to ensure the accuracy of the experimental data, the experiment was conducted twice in each condition, and four groups of tests were conducted. At the end of the entire experimental setup, a water tank was placed to recover the fragments and residual projectile. The overall arrangement of the experimental equipment is shown in Figure 8. 

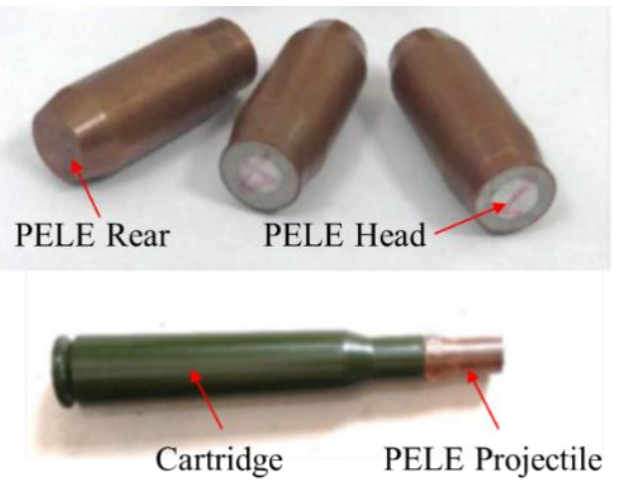

(a)

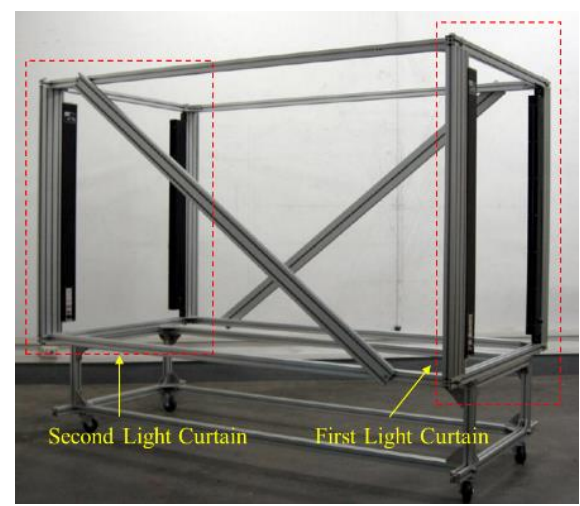

(c)

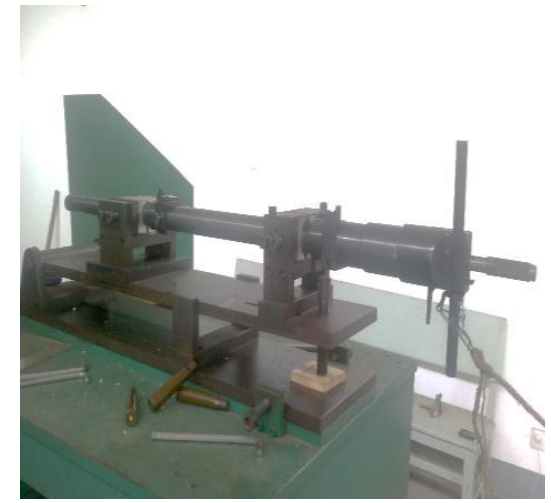

(b)

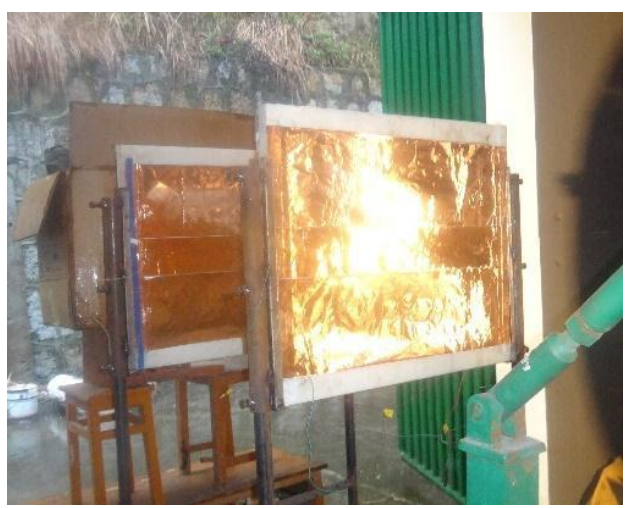

(d)

Figure 7. Different parts of the experimental apparatus. (a) Experimental projectile; (b) experimental gun; (c) light curtain target; (d) copper foil target.

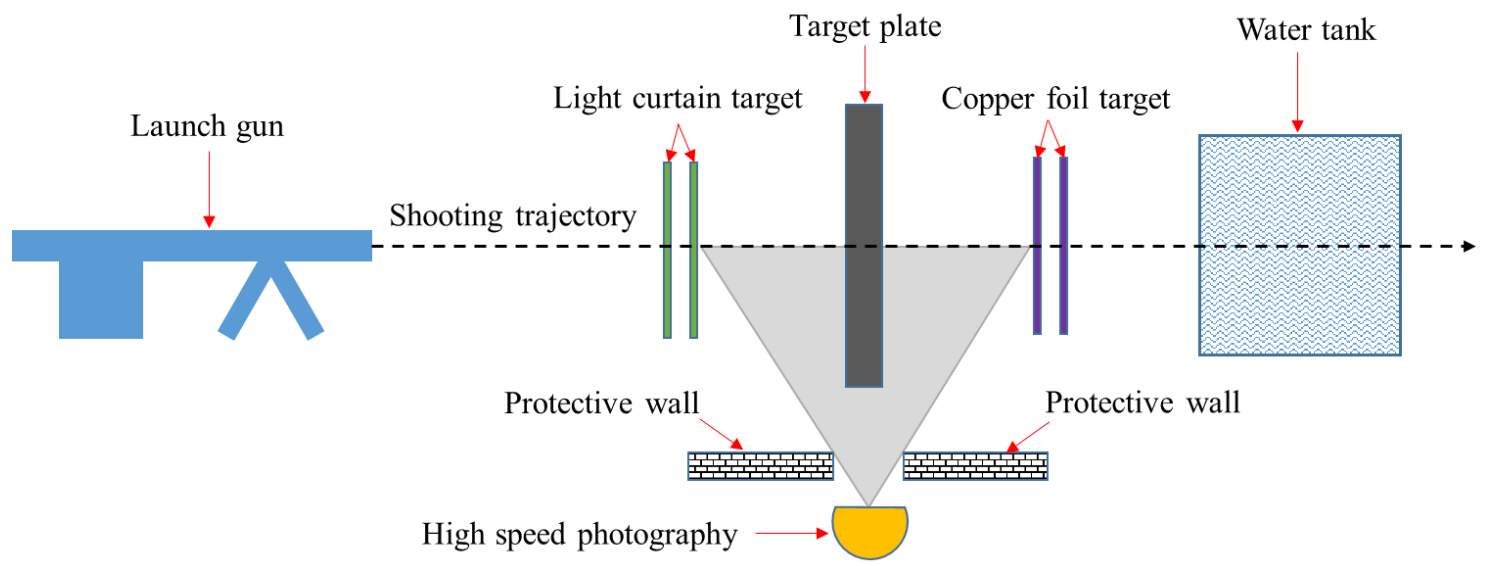

Figure 8. Experimental equipment layout diagram.

In order to more visually display the fragmentation effect of the PELE projectile after perforating the thin metal target plate, the fragments of the PELE projectile outer casing were collected from the water tank, as shown in Figure 9. Moreover, the quantity and total mass of recycled fragments were counted, as shown in Table 5. 


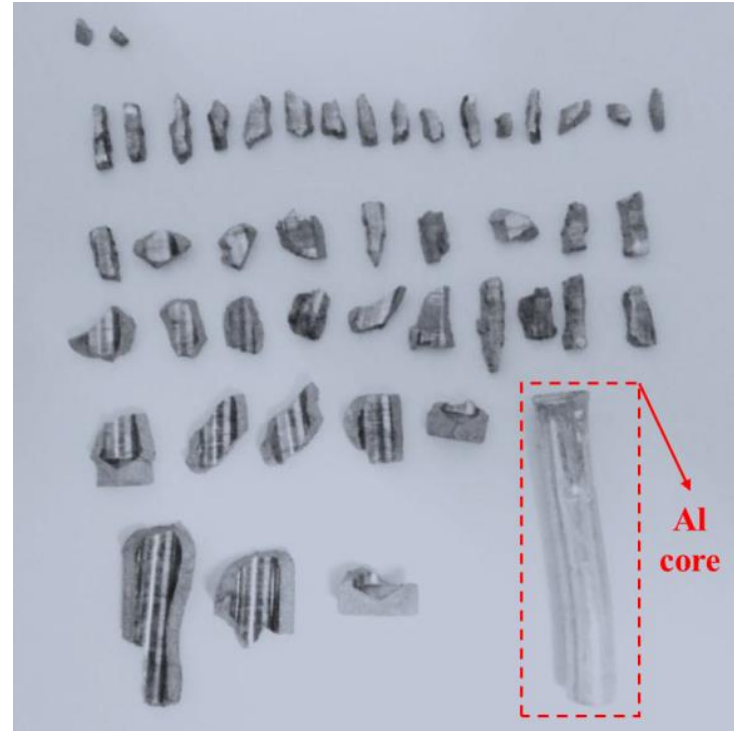

(a)

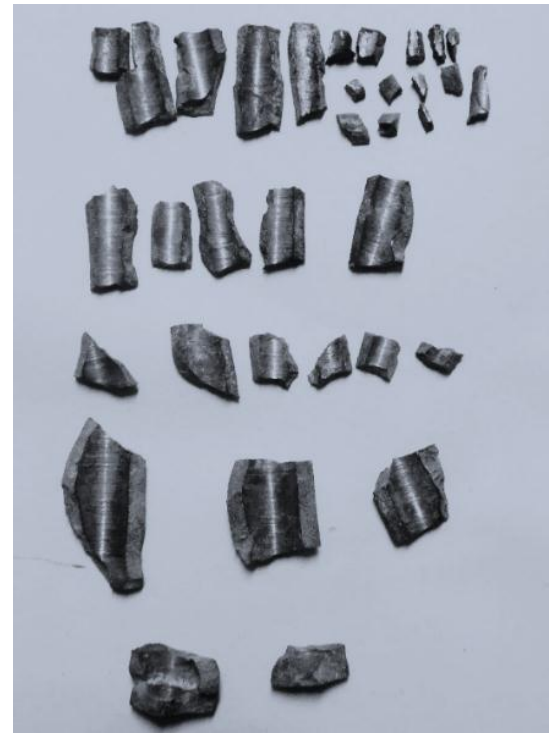

(b)

Figure 9. The fragments of PELE projectile outer casing collected from the water tank. (a) Al-1\#; (b) PTFE-1\#.

Table 5. Quantity and total mass of recycled fragments.

\begin{tabular}{ccccc}
\hline $\begin{array}{c}\text { Inner Core } \\
\text { Material }\end{array}$ & $\begin{array}{c}\text { Test } \\
\text { Number }\end{array}$ & $\begin{array}{c}\text { Quantity of } \\
\text { Recycled } \\
\text { Fragments }\end{array}$ & $\begin{array}{c}\text { Total Mass of } \\
\text { Recycled } \\
\text { Fragments }\end{array}$ & $\begin{array}{c}\text { Percentage of } \\
\text { Recovery Mass of } \\
\text { Outer Casing (\%) }\end{array}$ \\
\hline \multirow{2}{*}{ Al } & $1 \#$ & 45 & 34.0 & 90.2 \\
& $2 \#$ & 42 & 34.4 & 91.2 \\
\hline \multirow{2}{*}{ PTFE } & $1 \#$ & 34 & 31.7 & 84.1 \\
& $2 \#$ & 29 & 31.8 & 84.4 \\
\hline
\end{tabular}

From Figure 9, it can be seen that the PELE projectile was broken into a lot of fragments after perforating the target, the structure of the $\mathrm{Al}$ inner core was relatively complete, and plastic deformation occurred only in the head; the PTFE inner core was completely broken and the corresponding debris was not recovered in the water tank. Moreover, from the shape of fragments in Figure 9, the rupture of the projectile outer casing did not occur in the direction of thickness; only circumferential and axial rupture occurred. The above phenomena can well explain the difference between PELE projectiles and traditional armor piercing projectiles, that is, they possess a certain penetration ability and also have a certain lateral fragmentation effect.

Assuming that the time taken for the PELE projectile to pass through the two copper foil targets is $t$, and the distance between the two copper foil targets is $L_{0}$, the axial residual velocity of the projectile can be expressed as follows.

$$
u_{\text {res }}=\frac{L_{0}}{t}
$$

The theoretical and experimental results of the residual velocity of PELE projectiles are shown in Table 6 and Figure 10a. According to the relative error formula defined above, the relative error between the experiment results and the calculation results of the different inner cores is shown in Figure 10b. It can be obviously seen that the calculation results and the experimental results coincide well for the two kinds of PELE projectiles with different inner cores, which also illustrates the rationality of the theoretical model established in this paper. 
Table 6. Residual velocity of experiment and calculation for PTFE and Al core material.

\begin{tabular}{ccccc}
\hline $\begin{array}{c}\text { Inner Core } \\
\text { Material }\end{array}$ & $\begin{array}{c}\text { Test } \\
\text { Number }\end{array}$ & $\begin{array}{c}\text { Experimental } \\
\text { Initial Velocity } \\
(\mathbf{m} / \mathbf{s})\end{array}$ & $\begin{array}{c}\text { Experimental } \\
\text { Residual Velocity } \\
(\mathbf{m} / \mathbf{s})\end{array}$ & $\begin{array}{c}\text { Calculated } \\
\text { Residual Velocity } \\
(\mathbf{m} / \mathbf{s})\end{array}$ \\
\hline \multirow{2}{*}{$\mathrm{Al}$} & $1 \#$ & 806 & 788 & 779 \\
& $2 \#$ & 798 & 780 & 772 \\
\hline \multirow{2}{*}{ PTFE } & $1 \#$ & 809 & 790 & 784 \\
& $2 \#$ & 802 & 787 & 776 \\
\hline
\end{tabular}

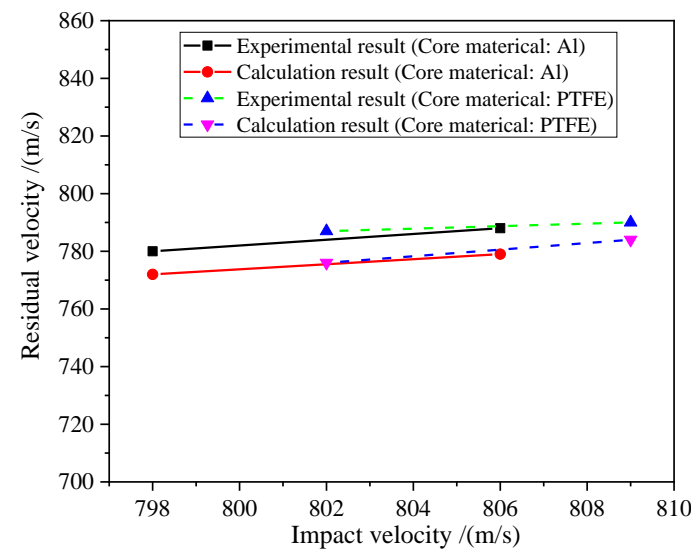

(a)

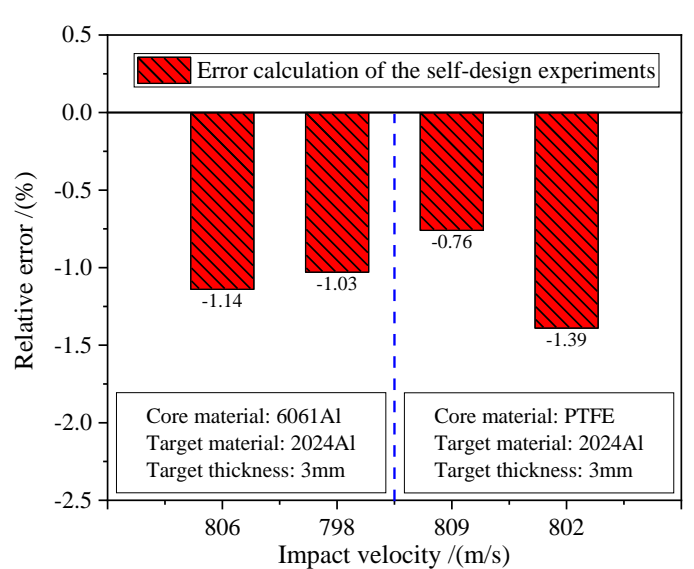

(b)

Figure 10. The residual velocity and relative error of experiment and calculation under different inner core material conditions. (a) Residual velocity; (b) relative error.

\section{Conclusions}

Since the PELE projectile is a symmetrical structure, the collision between the projectile and the target plate can be simplified to a plane collision problem. Moreover, because the two-dimensional plane is axisymmetric, it can be further simplified to one-dimensional collision. Based on shock wave theory, the shock wave discontinuous equation, and the linear shock equation, the theoretical model of the axial residual velocity of a PELE projectile penetrating thin metal targets was established. In order to verify the rationality of the theoretical model, we compared the calculation results obtained by the theoretical model with the experimental results in Reference [3] and the self-designed experimental results. The comparison results show that they are in good agreement, and we can also draw the following conclusions:

(1) Based on the experimental results in Reference [3], the theoretical model established in this paper can be well matched for both lower and higher velocities, but the calculated results based on the elastic wave theory used in Reference [3] were close to the experimental results at lower velocity. Therefore, the calculated results based on shock wave theory in this paper are better than those based on elastic wave theory in Reference [3], especially in high velocity conditions. Through comparative analysis, we confirmed the rationality of the theoretical model established in this paper.

(2) Four groups of tests were self-designed to further verify the theoretical model, and the impact velocity of the PELE projectile was about $800 \mathrm{~m} / \mathrm{s}$. The results show that the experimental results were in good agreement with the calculated results, and the error between them was within $1.5 \%$.

The above two verification methods all illustrate that the theoretical model established in this paper is scientific and reasonable. In addition, by comparing the theoretical results and experimental data in Reference [3], it was also found that when the projectile impact velocity is below $3000 \mathrm{~m} / \mathrm{s}$, the theoretical model established in this paper can be applied very well. 
Author Contributions: Conceptualization, L.D. and W.T.; methodology, X.R.; validation, L.D. and X.R.; formal analysis, Z.F.; resources, W.C.; data curation, Z.F.; writing-original draft preparation, L.D.; writing-review and editing, W.T.; supervision, W.C.; project administration, W.T.

Funding: This research was funded by the National Natural Science Foundation of China (grant Nos. 11002162 and 11072262).

Acknowledgments: The authors would like to thank the technical staff in the Minghua Machinery limited company of Guangdong, for their experiment supports.

Conflicts of Interest: The authors declare no conflict of interest.

\section{References}

1. Kesberg, G.; Schirm, V.; Kerk, S. PELE-The future ammunition concept. In Proceedings of the 21st International Symposium on Ballistics (ISB'21), Adelaide, Australia, 19-23 April 2004.

2. Paulus, G.; Chanteret, P.Y.; Wollmann, E. PELE: A new penetrator-concept for the generation of lateral effects. In Proceedings of the 21st International Symposium on Ballistics (ISB'21), Adelaide, Australia, 19-23 April 2004.

3. Paulus, G.; Schirm, V. Impact behavior of PELE projectiles perforating thin target plates. Int. J. Impact Eng. 2006, 33, 566-579. [CrossRef]

4. Zhu, J.; Zhao, G.; Du, Z. Numerical simulation and experimental research of PELE impacting target with different velocities. In Proceedings of the International Conference on Mechanical Engineering and Mechanics, Wuxi, China, 5-7 November 2007.

5. Jiang, J.; Zhang, M.; Men, J.; Wang, S. Experimental study on multi-layered target penetration of PELE with different cores. Trans. Beijing Inst. Technol. 2013, 33, 1009-1012.

6. Jiang, J.; Zhang, M.; Men, J.; Wang, S. Study on fragmentation of PELE against thin targets. Trans. Beijing Inst. Technol. 2011, 20, 168-172.

7. Ji, P.; Wang, H. Ballistic limit and residual velocity of PELE penetrating against metal target. Trans. Beijing Inst. Technol. 2011, 20, 183-186.

8. Ji, P.; Wang, H.; Zheng, Y.; Yu, Q. Influence of structure parameters on terminal effect of small caliber PELE impacting metal target. Trans. Beijing Inst. Technol. 2011, 20, 187-191.

9. Verreault, J. Analytical and numerical description of the PELE fragmentation upon impact with thin target plates. Int. J. Impact Eng. 2015, 76, 196-206. [CrossRef]

10. Wang, H.; Ji, P.; Yu, Q.; Zheng, Y. Numerical simulation of oblique penetration of PELE into finite thickness plates. Trans. Beijing Inst. Technol. 2010, 30, 1017-1019.

11. Arias, A.; Rodriguez-Martinez, J.A.; Rusinek, A. Numerical simulation of impact behavior of thin steel plates subjected to cylindrical, conical and hemispherical non-deformable projectiles. Eng. Fract. Mech. 2008, 75, 1635-1656. [CrossRef]

12. Zhu, J.; Zhao, G.; Du, Z. Influence of the filling material on the PELE effect. J. Ballist. 2007, 19, 62-65.

13. Zhu, J.; Zhao, G.; Du, Z.; Wang, X. Mechanism of PELE projectiles perpendicularly impacting on thin target plates. Explos. Shock Waves 2009, 29, 281-288.

14. Du, Z.; Song, L.; Zhong, K.; Wang, F. Influence of the ratio of inner to outer diameter on penetrator with enhanced lateral efficiency. J. Comput. Theor. Nanosci. 2011, 4, 1525-1528. [CrossRef]

15. Grady, D. Fragmentation of Rings and Shells: The Legacy of NF Mott; Springer: Berlin/Heidelberg, Germany, 2006.

16. Hirsch, E. The Mott fragmentation model and the Vpl break-up parameter. Propellants Explos. Pyrotech. 1989, 14, 31-38. [CrossRef]

17. Verreault, J.; Hinsberg, N.; Abadjieva, E. PELE fragmentation dynamics. In Proceedings of the 27th International Symposium on Ballistics (ISB'27), Freiburg, Germany, 22-26 April 2013.

18. Verreault, J. Modeling of the PELE fragmentation dynamics. In Proceedings of the 18th Biennial International Conference of the APS Topical Group on Shock Compression of Condensed Matter Held in Conjunction with the 24th Biennial International Conference of the International Association for the Advancement of High Pressure Science and Technology, Seattle, WA, USA, 7-12 July 2013.

19. Fan, Z.; Ran, X.; Tang, W.; Ke, Y.; Li, Z. The model to calculate the radial velocities of fragments after PELE perforating a thin plate. Int. J. Impact Eng. 2016, 95, 12-16. [CrossRef] 
20. Wang, X.; Zhu, X. Study on high-velocity blunt-nosed projectiles penetrating moderate thickness metallic targets. Eng. Mech. 2010, 27, 213-217.

21. Meyers, M. Dynamic Behavior of Materials; A Wiley-Interscience Publication: New York, NY, USA, 1994.

22. Century Dynamics Inc. Interactive Non-Linear Dynamic Analysis Software AUTODYN User Manual; Century Dynamics Inc.: Oakland, CA, USA, 2003. 